\title{
Improvement in Performance of Image Retrieval using Various Features in CBIR System
}

\author{
Dipesh Patel \\ ComputerDepartment \\ Sankalchand Patel College of Engineering, \\ Visnagar,India
}

\author{
Darshan Patel \\ ComputerDepartment \\ Sankalchand Patel College of Engineering, \\ Visnagar,India
}

\begin{abstract}
Content-Based image retrieval systems (CBIR) have become very popular for browsing, searching, and retrieving images from a large database of digital images as it requires relatively less human interference. In Content-based image retrieval system, visual feature. Color, texture and shape features have been the primitive image descriptors in CBIR systems. By using only color, texture or shape features, cannot get high precision. So, propose a new content-based image retrieval method that uses combination of color, shape and texture feature to get high precision. By using techniques like Image Processing, Data Mining, Machine Learning and Database for extracting color features, texture features and shape features, In this paper discuss the using various features and technique to possible get best precision as well as less computational complexity and good retrieval accuracy.
\end{abstract}

\section{Keywords}

Data Mining, Image Mining, Content Based Image Retrieval, Feature extraction, Image retrieval.

\section{INTRODUCTION}

Now a Day's peoples are covered by lots of Data medical data, financial data, scientific data, geographic data and marketing data. Peoples have no time to look at this data, so must need to find ways to automatically analysis and classify data. In current environment capacity of generating and collecting data has been increasing rapidly, with the help of computerization business, scientific research and government transaction, digital camera, barcodes etc. [1]

The lots of amount of data and information are stored in World Wide Web (WWW) the amount of this data and information growing very quickly. so an argent need for new techniques and automated tool is generated which can intelligently assist us in transforming the large amount of data in to useful information and knowledge hence the new term data mining is introduce data mining refers to extracting or "mining" knowledge from large amount of data many other term carry a similar or slightly different meaning to data mining such as knowledge mining from data, knowledge extraction data/pattern analysis etc.[1]

There are major research area in data mining including (a) associate mining (b) image mining (c) web mining (d) text mining (e) clustering data mining (f) spatial data mining etc.The rest of this paper is organized as follows. Section 2 presents the Background terminology. Section 3 presents the Related work or existing CBIR work. Section 4 proposed method and Section 5 presents the Conclusion and future work related with this research.

\section{BACKGROUND}

In this Section classify Image mining word, Image mining is most important techniques which is used to mine knowledge straightforwardly from image in advance, image segmentation is primary phase of image mining.[2] Advances in image acquisition and storage technology have led to tremendous growth in significantly large and detailed image databases. Image mining deal with the extraction of knowledge, image data relationship or other pattern database. The focus of using image processing techniques is extracting specific features from a single image.[3]

Image mining is some method allow image mining have to different approaches first method is extract image in image database or huge or collection of image. Second method is more deep mined the combination of associated alphanumeric data and collection of image. Image mining is an expansion of data mining in field of image processing image mining handle with a much of hidden knowledge extraction, image data association pattern. Research area in image mining can be broadly classifying in two factor (a) Domain specific application and (b) General application. Both factor used to extract to image features and after processing to generate image pattern. $[3,4]$

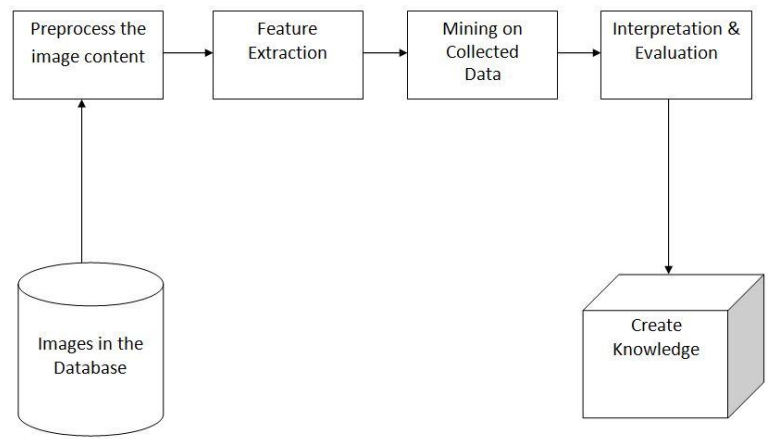

Fig 1: Image Mining Process [5]

Show in the fig. 1 image mining process image is retrieved in to the collection of images database first step is retrieved image on apply the preprocessing to improve quality then after next phase on image feature extraction to important feature from images generated features after using different data mining techniques to discover significant patterns. This resulting pattern to evaluate and obtain final knowledge retrieved.[5]

"Image retrieval" means that searching and browsing aims at retrieving images from a large database of digital images. Many of Images database (DB) is traditional type of database. in traditional method of image annotation is time consuming, laborious and expensive in this old method is use pointing image by indexing, it means of storing image in image database that time associated it with image keyword, tag or number like it called metadata show in fig. 2 is image retrieval model. 


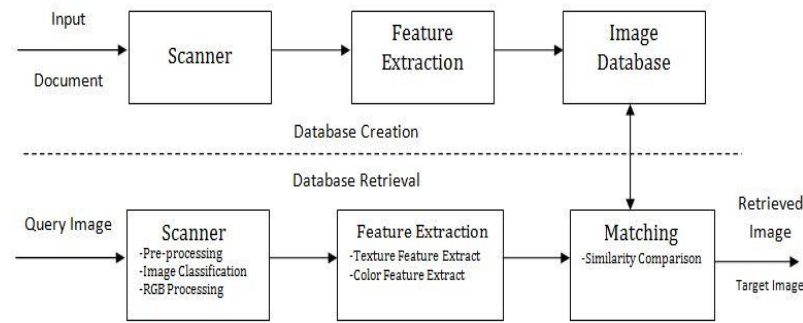

Fig.2: Image Retrieval System Flow [6]

Here fig. 2 shows that the phase Database creation and database retrieval phase in first phase on image input by using query system than first of scan the image surface in image on several of content and feature this feature extraction and store in image database. another side is database retrieval phase in this phase on same step first of image input or query image than perform in scanning stage on this stage various of technique and method apply to improvement quality using pre-processing, image classification and RGB processing on image pass on next stage in feature extraction in this stage image on various kind of feature store in image like color, texture, shapes feature extracting and last stage on matching feature with existing feature store database if matching similarity than retrieved image is call target image.[6].

There are two fundamental type of image retrieval system.[6]
(a) Text Based Image Retrieval(TBIR)
(b) Content Based Image Retrieval(CBIR)

\subsection{Content-Based Image Retrieval System(CBIR)}

First of Text Based Image Retrieval (TBIR) system is traditional technique of retrieval and stored image based on keyword wise. Content Based Image Retrieval System (CBIR) is introduced on early 1990 .content base image retrieval system is techniques of retrieving image based on their various features such as colour, texture and shapes. Content based image retrieval system (CBIR) is also known as Query by image content (QBIC) and content-based visual information retrieval (CBBIR) the advantage of CBIR system are that is a fast method and able to extract the low-level feature automatically. This low level feature such as colour, texture, shapes and extract from image for measuring the similarity between different images and for retrieving similar images.[7,8].Content Based Image Retrieval is extremely useful in a application such as Faison geographical information architectural and engineering design medical diagnosis etc.[14]

Show in CBIR System architecture fig. 3 in it is divided in to three parts such as interface, query processing module, and image database. while inserting a new image in a database, first the feature of the image like color, texture and shapes are extracted from the image and stored along with image in image database for while retrieving the related images based on one image first of all the feature extracted from that image and compared with the feature which are already stored in database if the similar features are an-counted then that images are feather from database and directly given to the target application.

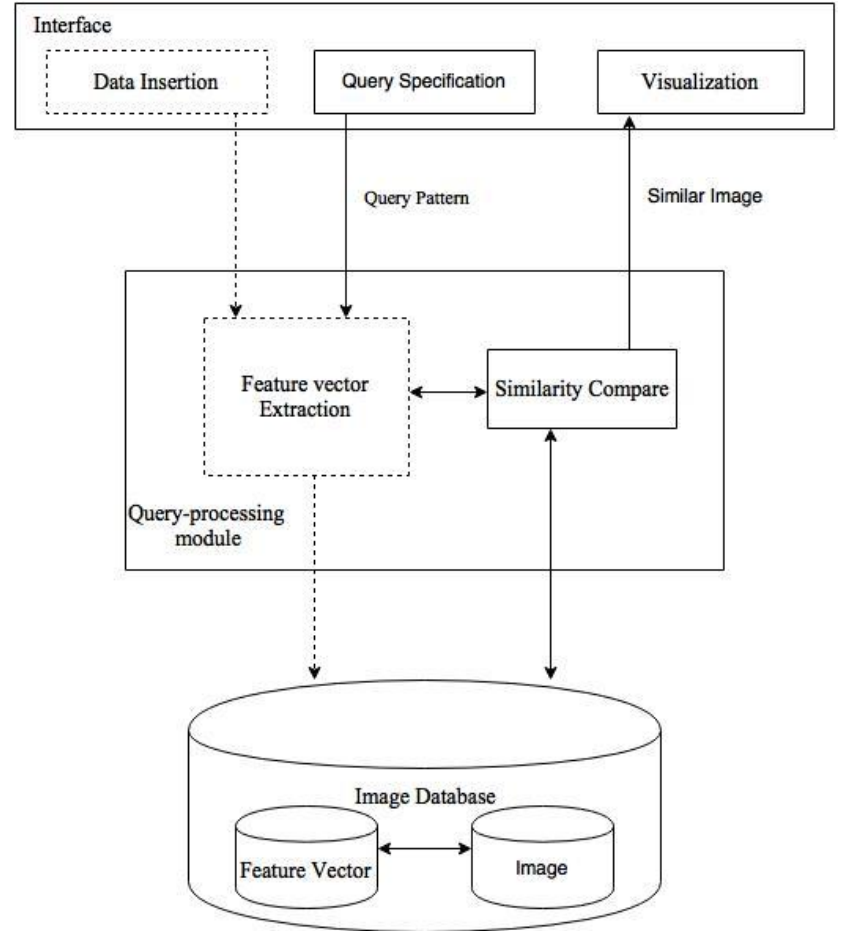

Fig 3: CBIR System Architecture [9]

\subsubsection{Color Feature:}

Color Feature is an important characteristics of the content based image retrieval system. Color are used in image processing because they are provide powerful descriptor that can be used to identify and extract object from a image.[14].color model based on hue, saturation and lightness (HSV) or also primary properties is Red, Green and Blue (RGB).Many method can be used to describe color feature it is color histogram, color correlation, color moments etc. here describe color extraction using mathematically of mean, standard deviation and skewness it is color moment providing to be color on image is effective and efficient result providing.[14]

Formula 1 Mean:

$$
E_{i}=\frac{1}{N} \sum_{j=1}^{\mathrm{N}} \mathrm{p}_{\mathrm{ij}}
$$

Formula 2 Standard Deviation:

$$
\sigma_{i}=\sqrt{\left(\frac{1}{N} \sum_{j=1}^{N}\left(p_{i j}-E_{i}\right)^{2}\right)}
$$

Formula 3Skewness:

$$
S_{i}=\sqrt[3]{\left(\frac{1}{N} \sum_{j=1}^{N}\left(p_{i j}-E_{i}\right)^{3}\right)}
$$

\subsubsection{Texture Feature:}

Image texture can be seen on area of image contain in repeated pattern on pixel extremely arranged in some structural way.[10].In image contain important feature or information about structural arrangement of surface and their relationship.[11].More texture display in the natural type of 
images as in clouds, tree, brick walls and grasslands etc. Gray Level Co-occurrence Matrix (GLCM) has proved to be accurate method of extracting textural feature from images.[12] Texture feature measure the based on properties such as Smoothness, Coarseness, Roughness, Regularity, Directional, line likeness etc.[14] Ranklet transform is family of multiscale,non-parameters feature modeled.[13] Ranklet transform apply before you extract the texture feature than the apply preprocessing step using ranklet transform. When you apply ranklet transform on image is three-direction (side) (vertical, horizontal, diagonal) on apply ranklet transform.[14].here some of example of texture features show in fig.4.

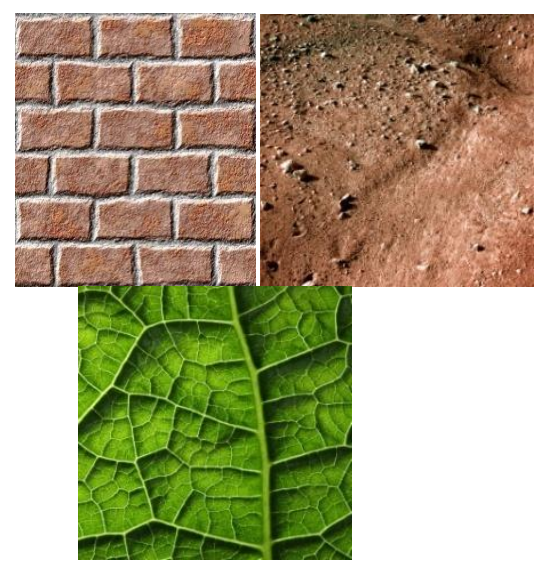

(a)

(b)

(c)

Fig 4: Texture Feature Example.

\section{RELATED WORK}

This section gives a brief review about the features extracting using the Content Based Image Retrieval system.

Ahmed J. Afifi and Wesam M. Ashour et al.[14] used an algorithm called Color feature and ranklet transform is used. This algorithm used to various of features extraction in to image and more accurate result is retrieved. The problem identify is Extract color features and texture features from image the issue is complexity of image data to the image database.

M.E. ElAlami et al.[15] proposed an algorithm known as Color Features\& Texture Features \& ANN it is accurate and rapid model for con-tent based image retrieval process depending on a new matching strategy. Problems are using a single feature, then the system works slowly. Disadvantages are a lot of training of same type of images has to be given for achieving accuracy.

A. A. Khodaskar, S. A. Ladhake et al.[16] proposed an ANN, Fuzzy Logic, SVM its is soft computing based techniques .In image database environment lot of processing time and high amount of training will be required.

Roshi Choudhary et al.[17] present an algorithm is Color Features \& Texture Features using Local Binary Patterns (LBP) . To extract the color feature, color moment $(\mathrm{CM})$ is used on color images and to extract the texture feature, local binary pattern (LBP) is performed on the grayscale image. Main drawback is use only one feature to perform on retrieval image then retrieval result is not accurate.

Sudeep D. Thepade, Yogita D et al.[18] proposed algorithm advantages is Fri-chen and sobel it is improving performance in
CBIR using color edge detection. some major issue in current environment in image retrieval in to image database the precision of query execute time is more it means of issue is two are performance and accuracy are major problem.

\section{OUR CONTRIBUTION}

The work flow of system proposed by AhmdJ.Afif [14] is as follow for retrieving similar images query is generated using one image then the features like color, texture and shapes are extracted from that image and matched with the features which are already stored in the database if successfully match is found than that related images are retrieved from the database.

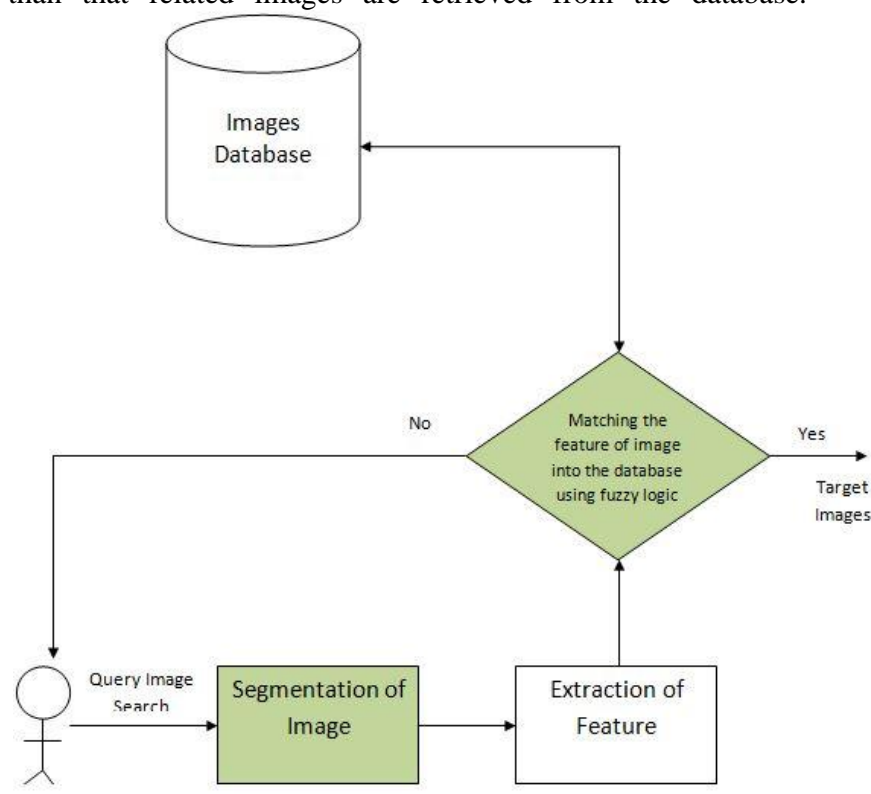

Fig 5: Proposed Work[14]

Here, contribution has been illustrated by green boxes. In which added two techniques one segmentation of image, second fuzzy logic which are briefly describe as follow (a) segmentation of image in this technique the whole image is divided into small segment the segmentation of image done in two phase, first horizontal segmentation and vertical segmentation by using this segmentation technique get more better feature from the image (b) and using fuzzy logic get better accuracy while matching the features of the query image and the features already stored in the database so can get only necessary images which are trying to retrieved.

Proposed System Workflow:

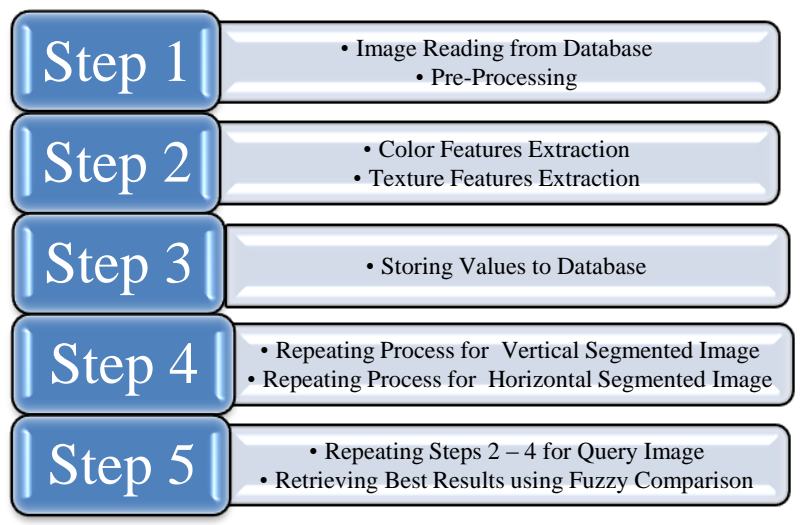

Fig 6: Proposed System Workflow 
Performance measured in CBIR system using by two factors Precision and Recall. Precision is ration of the number of relevant image retrieved and total number of image retrieved. Precision is denoted by $\mathrm{P}$.

Precision $(\mathrm{P})=\frac{\text { Number of Relevant image retrieved }}{\text { Total number of image retrieved }}$

Recall $(R)=\frac{\text { Number of Relevant image retrieved }}{\text { Total number of relevant images in }}$ the database

Second, factor is recall is ratio of number of relevant image retrieved and total number of image retrieved.

\section{CONCLUSION AND FUTURE WORK}

Content Based Image Retrieval (CBIR) system is the major issue in Image Mining. Although CBIR has been a very current research are since 1990's.many of research done on content based image retrieval for minimizing of complexity and getting better output but most of them not up to the mark.

Purpose of this survey paper is to finding the techniques which helps to improve the accuracy while retrieving the images from image database. In feature use two techniques is segmentation of image-which will helps for better feature extraction and fuzzy logic while gives better accuracy while matching image features.

\section{REFERENCES}

[1] Jiawei Han University of Illinois at Urbana-Champaign MichelineKamber, "Data Mining Concepts and Techniques.2nd.

[2] A.Hema1, E.Annasaro2Nuaimi, "A Survey in need of image mining techniques" (February 2013).International Journal of Advanced Research in Computer and Communication Engineering Vol. 2, Issue 2, February 2013 page $1238-1240$

[3] JiZhang,WynneHsu,Mong Li Lee "Image minig: Issue,Framework and techniques"

[4] RamadassSudhir, "A Survey on Image Mining Techniques: Theory and Applications" Computer Engineering and Intelligent Systems ISSN 2222-1719 (Paper) ISSN 2222-2863 (Online)Vol 2, No.6, 2011

[5] A.Kannan, Dr.V.Mohan, Dr.N.Anbazhagan "An Effective Method of Image Retrieval using Image Mining Techniques" (November 2010).The International journal of Multimedia \& Its Applications (IJMA) Vol.2, No.4.

[6] Monika Sahu, MadhupShrivastava "Image mining: A new approach for data mining based on texture"2012 Third
International Conference on Computer and Communication Technology IEEE page 7-9

[7] S.Pradeep, Mrs.L.Malliga "Content based image retrieval and segmentation of medical image database with fuzzy" 2014 IEEE ICICES 2014

[8] Ahsan Raza Sheikh, SarinaMansor, Mohd. H. Lye ,Mohd. F. A. Fauzi“"Content Based Image Retrieval System for Marine Life Images using Gradient Vextore Flow " 2013 IEEE International Conference on Signal and Image Processing Applications (ICSIPA)

[9] Ricardo da Silva Torres, Alexandre Xavier Falcão"Content-Based Image Retrieval: Theory and Applications" 2006 Institute of Computing, State University of Campinas, Campinas, SP, Brazil. Volume XIII, page 165-189

[10] Nadia Baaziz, Omar Abahmane and RokiaMissaoui "Texture feature extraction in the spatial-frequency domain for content-based image retrieval".

[11] Vivek Jain, NehaSahu "A Survey: On Content Based Image Retrieval”Vol. 3, Issue 4, Jul-Aug 2013, pp.11661169

[12] S.Asha, S.Bhuvana, Dr.R.Radhakrishnan"A Survey on Content Based Image Retrieval Based on Feature Extraction” 2014 Vol 1, Issue 06;pp 29-34

[13] ManasSaha, Snigdhadeb Roy Chowdhury, Kyamelia Roy "Ranklets: A Qualitative Review" September 2011pp 68 73.

[14] Ahmed J. Afifi, Wesam M. Ashour, "Content-Based Image Retrieval Using Invariant Color and Texture Features", IEEE 2012

[15] M.E. ElAlami, "A new matching strategy for content based image retrieval system", Elsevier October 2013

[16] A.A. Khodaskar, S. A. Ladhake, "A Novel Approach for Content Based Image Retrieval in context of combination S C Techniques", IEEE October 2015

[17] RoshiChoudhary, Nikita Raina, Neeshu Chaudhary, Rashmi Chauhan, Dr. R H Goudar, "An Integrated Approach to Content Based Image Retrieval”, IEEE 2014.

[18] Sudeep D. Thepade, Yogita D. Shinde "Improvisation of Content Based Image retrieval using Color Edge Detection with various Gradient Filters and Slope Magnitude Method" 2015 International Conference on Computing Communication Control and Automation 2015-IE 